\title{
Biofilm control by ionic liquids
}

Manuel Simões', mvs@fe.up.pt, Ana Rita Pereira', Lúcia Chaves Simões ${ }^{2}$, Fernando Cagide ${ }^{3}$ and Fernanda Borges ${ }^{3}$, fborges@fc.up.pt

Ionic liquids (ILs) are remarkable chemical compounds with applications in many areas of modern science. They are increasingly recognized as promising compounds to fight microorganisms in both planktonic and biofilm states, contributing to reinvent the antimicrobial pipeline. Biofilm-related infections are particularly challenging given that the scientific community has not yet identified a reliable control strategy. Understanding of the action of ILs in biofilm control is is still in a very early stage. However, given the highly tunable nature and exceptional properties of ILs, they are excellent candidates for biofilm control. Here, we review the major advances in, and challenges tothe use of ILs for effective biofilm control.

\section{Introduction}

Microorganisms are typically found adhered on biotic or abiotic surfaces as complex sessile communities embedded in a matrix of extracellular polymeric substances (EPS) that they produce $[1,2]$. According to the National Institute of Health, it is estimated that $80 \%$ of microbial infections in humans are biofilm related and affect 1.4 million people annually [3], promoting disease transmission, prolonged hospitalization and additional clinical procedures, thus increasing healthcare costs, and mortality and morbidity rates $[4,5]$. Most bacterial-associated infections, including endocarditis, dental caries, middle ear infections, osteomyelitis, medical device-related infections, and chronic lung infections in patients with cystic fibrosis, are problematic or untreatable because of biofilms. The pathological process of biofilm formation in human infections is a multifactorial event that can be divided in four main steps (Fig. 1) [6].
The presence of biofilms is associated with the failure of conventional antimicrobial therapeutic approaches, with antibiotic resistance being of particular concern when bacteria form biofilms. Antibiotic concentrations necessary to inhibit bacterial biofilms can be up to $10-1000$ times higher than those needed to inhibit the same bacteria grown planktonically [7]. In addition to resistance mechanisms found in planktonic cells (gene transfer from resistant counterparts, efflux pumps, cellular impermeability imparted by the outer layers, enzymes that confer resistance, and natural evolutionary mutations) [8], there are several mechanistic hypothesis to explain the increased resistance of biofilms to antimicrobials: (i) direct interactions between the biofilm EPS and antimicrobials, affecting diffusion and availability; (ii) existence of an altered chemical microenvironment within the biofilm leading to areas of reduced or no growth (dormant cells); (iii) development of biofilm-specific phenotypes; (iv) ability of microorganisms in biofilms to express specific resistance genes; (v) possibility of damaged bacterial cells undergoing programmed cell death; and (vi) existence of persister cells $[2,7]$. With a dearth of new antibiotic and/or antibiofilm agents in the pipeline and ever-decreasing pharmaceutical industry involvement in the discovery of new drugs, there is a pressing need to find novel and innovative solutions.

ILs are increasingly recognized as relevant for antimicrobial purposes [9-13]. They are generally referred to as 'green solvents' because they fulfill all the 12 principles of Green Chemistry laid down by Paul Anastas and John Warner [14]. The ability to tune the physical, chemical, and biological properties of ILs by independent modification of the properties of each constituent (cationic head and tail, and anion), has been the major driving force behind the interest in their biomedical applications. Moreover, ILs have also been at the cutting edge for the development of prodrugs or drug delivery 


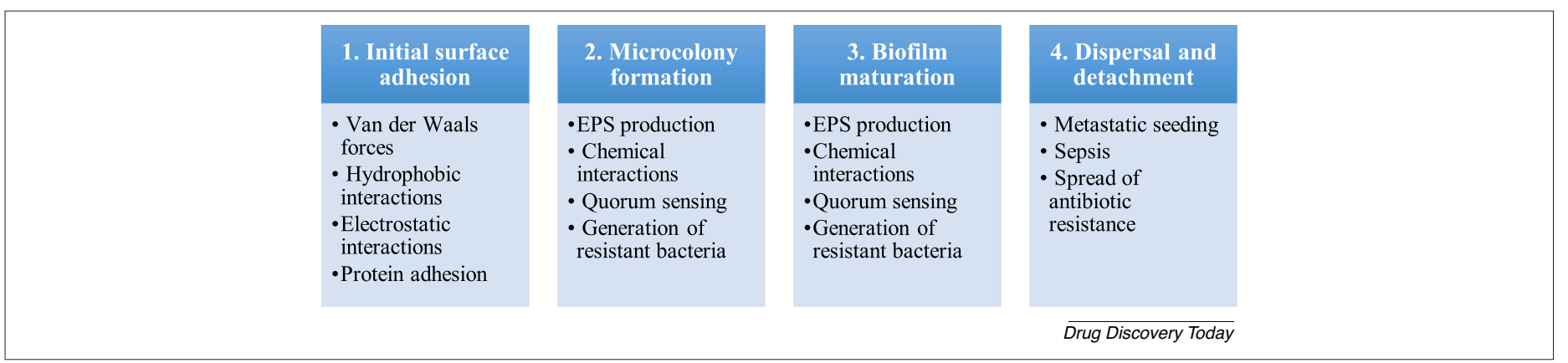

FIGURE 1

The main steps and processes involved in the development of infectious biofilms. Classically, four steps are considered: (1) initial adhesion followed by (2) microcolony formation, in which chemical interactions and signaling pathways are dominant allowing the formation of the matrix of extracellular polymeric substances (EPS). At that step, antimicrobial resistant bacteria appear as a consequence of the microcolony structure and composition. (3) Biofilm maturation is characterized by the formation of a 3D structure, where the EPS production, chemical signaling events, and the generation of resistant bacteria occur to a high extent, leading to the formation of recalcitrant infections. (4) As a biofilm matures, detachment events also occur, spreading cells and fragments of the biofilm to other regions. These cells and fragments can adhere and form a new biofilm, ultimately causing sepsis.

systems to solve problems related to solubility and permeability issues $[15,16]$. ILs were also found to be able to dissolve complex biopolymers [17], an effect of potential interest when searching for a strategy to disrupt the complex EPS matrix of biofilms. Here, we review the major achievements in, and challenges to the use of ILS as a new generation of therapeutic agents for effective biofilm control.

\section{Ionic liquids: an overview}

ILs are molten salts (most of them melt below $100^{\circ} \mathrm{C}$ ) consisting entirely of charged species (ions), with most being liquid at room temperature [18-20]. Recent reviews focused on recent advances in IL synthesis, organization, and modulation of physicochemical properties were published [15,21-24]. Currently, ILs are categorized in different generations, with the first and second-generation IL generally focused on modulation of physicochemical properties and applications, respectively. Third-generation ILs (advanced or task-specific ILs) were based on the use of biodegradable and natural ions or ions with known pharmacological activity, such as active pharmaceutical ingredients (API)
$[9,15,22,25]$. The exploitation of ILs relevant chemical space is currently of interest for biomedical and pharmaceutical applications.

The versatile properties of ILs, in conjunction with their potential for multiple functionalities, provide a myriad of innovative antimicrobial strategies with the chemical diversity needed for antimicrobial drug discovery programs and to progress the control of antimicrobial-resistant microorganisms [25]. ILs antimicrobial properties fully depend on their structure, which can be adjusted by altering the cationic organic head (positive charge usually located on the nitrogen

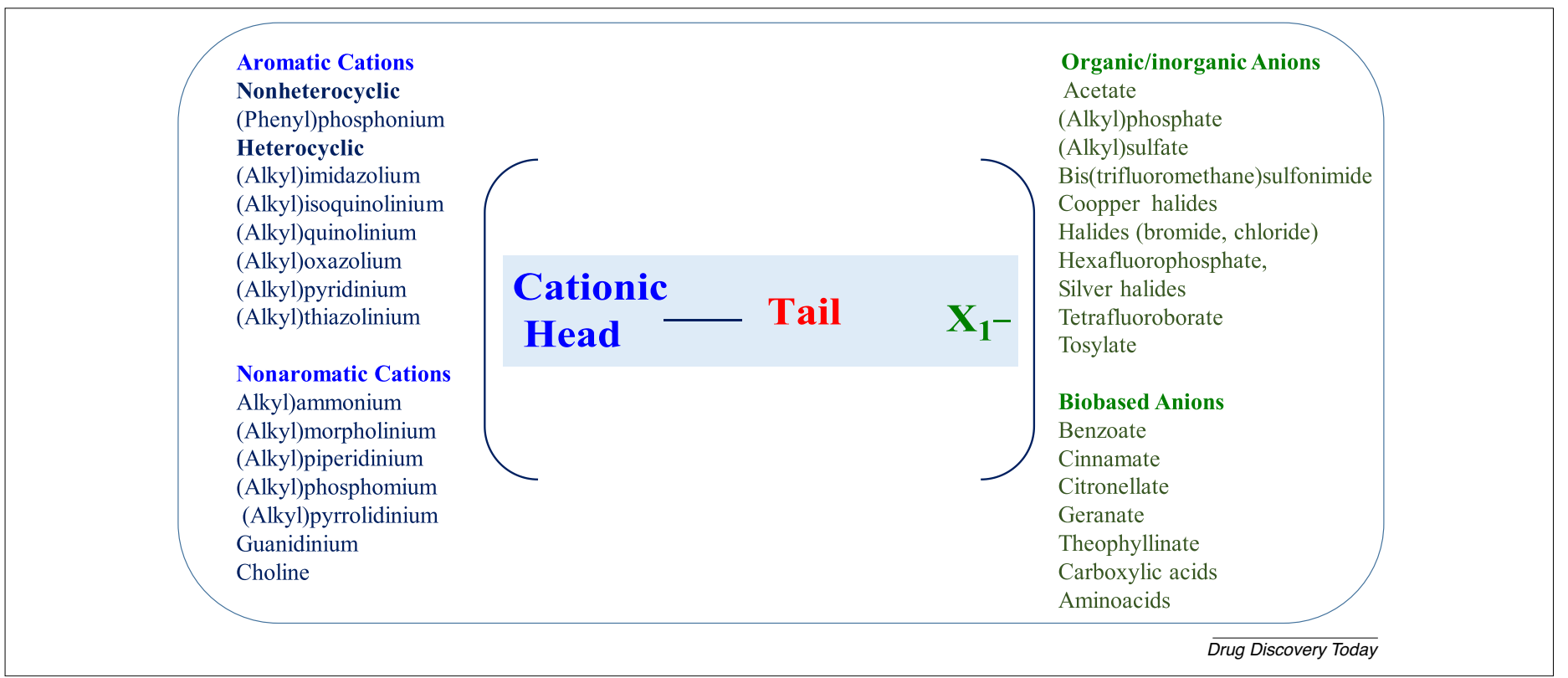

\section{FIGURE 2}

Most commonly described cations and anions of ionic liquids (ILs) used in biomedical studies. IL cations are categorized into two main types: aromatic (nonheterocyclic and heterocyclic) and nonaromatic; the anions are organic or inorganic and biobased. The tail is usually an alkyl linear chain of variable length. The chemical structures of each system are provided elsewhere $[9,15,21-26]$. 
or phosphorus atoms) the tail (usually an alkyl linear chain with a variable length) and the organic/inorganic or biobased anion (Fig. 2) $[21,26]$. The enormous range of cation-anion combinations, with some estimates as high as $10^{18}$ when in multiphase mixtures, results in a large potential for the adjustability of IL structure and properties $[27,28]$. The cations and anions of ILs commonly used in biomedical and pharmaceutical studies are described in Fig. 2.

\section{Ionic liquids as antimicrobials}

Generally, ILs display antimicrobial activity toward a range of bacteria as well as mycobacteria and fungi. However, the highest activity of ILs is seen in relation to Gram-positive cocci, such as Staphylococcus and Streptococcus and other Gram-positive bacteria, including Lactobacillus and Bacillus subtilis [25]. The biocidal properties of ILs is defined by the molecular architecture of each one that is mainly dictated by the structure of the cationand in particular by the length of the aliphatic alkyl chains (Fig. 2) [9].

The most common IL cationic head groups include imidazolium, pyridinium, quinolinium, morpholinium, pyrrolidinium, and choline ions (Fig. 2) [9]. Nonaromatic ILs are usually less toxic than those containing heterocyclic nitrogen rings [29-32]. In general, their antimicrobial activity and toxicity profile can be modulated via the introduction of substituents on the cationic head [33]. Quinolinium ILs are generally more potent than imidazolium ILs [34], with 1-alkylquinolinium ILs being one of the most potent antimicrobial ILs $[9,35]$. Relevant effects on the activity were found when a tail is present in the IL system (Fig. 2). An increase in chain length by extending the number of alkyl groups can increase the IL-cell surface affinity [36] and enhance their antimicrobial activity. A higher hydrophobicity of a surface-active compound results in a lower concentration needed for microbial growth control [37].

The most effective antimicrobial effects have been reported for ILs containing alkyl chains with 10-16 carbon atoms. The data showed that a balance between hydrophilicity and lipophilicity is crucial for the antimicrobial performance $[15,38]$. Quinoline-based ILs, such as the 1alkylquinolinium ILs, showed the highest antimicrobial activity when the alkyl chain was $12-$ 14-carbons long [39]. Pyrrolidinium ILs containing an alkyl chain with 14 carbon atoms display the highest antimicrobial activity against bacteria and fungi [31]. Nevertheless, the performance of ILs is strongly dependent on the type of cation because it has a significant role in determining the hydrophilic-lipophilic balance required. For example, trihexyl(alkyl)phosphonium ILs with an alkyl chain length of eight carbons are effective antimicrobials because they are able to easily penetrate the peptidoglycan cell wall of Gram-positive bacteria [40].

Although both cation and anions (Fig. 2) intrinsic to ILs can have inherent antimicrobial activity, varying the anion for the same cation does not appear to affect their outline [31]. However, for ILs with small organic cations, the chaotropicity of the anion can be a major contributor to their antimicrobial action because anions are typically chemical penetration enhancers $[41,42]$. This phenomenon was observed for some phosphonium-based ILs [43].

Another approach of potential interest is the use of amino acids (e.g., proline, tryptophan, phenylalanine, methionine, and valine) as anions [9]. ILs with imidazolium cations and tryptophan anions demonstrated potent antibacterial activity against both Gram-negative and Gram-positive bacteria [44]. Within this framework, the choline-geranate ILs (Fig. 2) are gaining interest because of their excellent antimicrobial activity [42]

Dicationic ILs (DILs) comprise two head groups (cations) linked by a rigid or flexible spacer and two anions. They are usually classified as homoanionic and heteroanionic dicationic ILs. DILs have a high ionic character and their properties can be tuned by changing the length and type of spacer as well as the type of

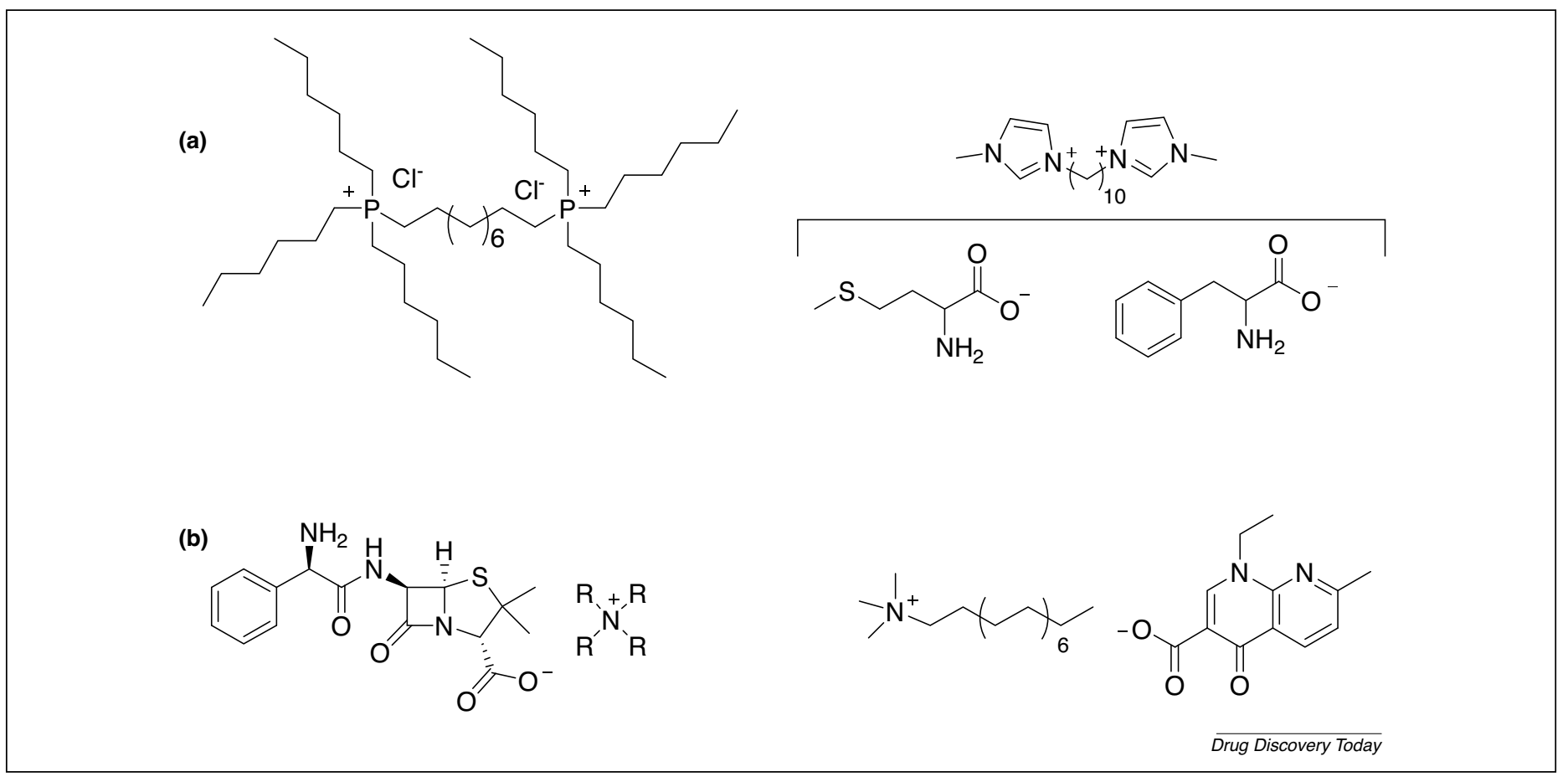

\section{FIGURE 3}

Examples of (a) dicationic ionic liquids (DILs) containing phosphonium and imidazolium cations and (b) dual-active salts containing antibiotics (ampicillin and nalidixic acid) and quaternary ammonium ILs. 
cation/anion. DILs have attracted increasing interest as antimicrobials because their hydrophobicity, efficacy,/selectivity, and toxicity can be tailored [15,28,45-47]. In fact, DILs containing imidazolium, benzimidazolium, pyridinium, ammonium and phosphonium counter cations have been described as potential antimicrobial agents. Examples of DILs used in antimicrobial studies are shown in Fig. 3a [48].

\section{Ionic liquids and drug delivery}

The use of ILs for drug delivery purposes has been recently reviewed [15,16,49-51]. Generally, they are used to modify drug pharmacokinetic properties, such as solubility, stability, and/or permeability across biological membranes, or in formulations.

Monocationic ILs are used carriers to increase the efficiency and reduce adverse effects of antimicrobial drugs. They typically work as bacterial membrane permeabilizers. There are several successful examples from the use of synergistic permeation enhancers, such as 1octyl-3-methylimidazolium-based ILs, choline and terpene-bioinspired ILs, and amine-based ILs, among others [52]. However, there is little published information available related to the application of dicationic ILs, and their benefits, in drug delivery. Based on this concept, and inspired by IL chemistry, dual-active API-ILs have also been developed. In this regard, active antimicrobial drugs can act as anion or cation moieties and ILs as the counter ions. The dual effect of API-ILs is dependent on the hydrophilicity/hydrophobicity of the counter ion, which can be used as a modulating factor for tuning the solubility and activity against various bacterial strains $[53,54]$. The API-IL concept has been extended to improve the performance of several antibiotics, such as ampicillin, ciprofloxacin, norfloxacin, nalidixic acid, penicillin G, amoxicillin, colistin, and antimicrobial peptides. The combination of 1-butyl-3methylimidazolium chloride and 1-butyl-3methylimidazolium tetrafluoroborate with polymyxin B enhanced bacterial membrane disruption and, thus- the antibiotic efficacy [55]. The most studied is the combination of ampicillin with quaternary ammonium ILs (Fig. 3b) [35]. Ampicillin has also been successfully paired with various organic cations, such as imidazolium, pyridinium, and choline [56]. Generally, this type of API-ILs are more effective against Gram-positive than Gram-negative bacteria,because of the absence of an outer membrane in the former, and can decrease the ampicillin concentration required for the antimicrobial effects [35].
The use of nano-based ILs approaches to develop drugdelivery systems by nanoencapsulation of antimicrobial drugs is limited. Thus far, ILs have been used to functionalize or coat silver, gold, and zinc oxide nanoparticles (NPs). This type of NPs demonstrated to be synergic in terms of antimicrobial activity against diverse bacteria [39,57-60]. Such combinations have a high surface area:volume ratio and unique chemical, physical, and antimicrobial properties [61]. In addition, nano-assembled systems, such as those based on phosphonium salts, have been used with positive outcomes [62].

\section{Antimicrobial mode of action of ionic liquids}

Antimicrobial ILs share structural and mechanistic analogies with cationic biocides, such as quaternary ammonium compounds (QACs), the primary mode of action of which is membranebound protein disruption [31,63]. In effect, mechanistic studies have shown that ILs target mainly the cytoplasmic (inner) membrane in bacteria and the plasma membrane in yeast [37], which leads to changes in the structural and dynamic properties of the outer layers [25] and subsequent disruption and loss of membrane integrity [64]. The general mechanism of action is based on the fact that most bacterial cells are negatively charged, often stabilized by the presence of divalent cations, such as $\mathrm{Ca}^{2+}$ and $\mathrm{Mg}^{2+}$, and that antimicrobial ILs are positively charged [65]. Therefore, during the first stage of antimicrobial action, there is an interaction between the cationic head group of ILs and the negative structural proteins of the bacterial outer layers [66]. Then, cations are readily absorbed into the cell layers, connecting to the cytoplasmatic membrane, and penetrating the interior of the cell [67]. ILs further provoke the coagulation of cytoplasmic constituents causing the inhibition of crucial enzymes (i.e., acetylcholinesterase, AMP deaminase, acylase I, cytochrome c oxidase, glutathione reductase, carboxylesterase, catalase, and Taq DNA polymerase), interfering with energy or self-repair processes $[68,69]$, and finally causing cell death [38].

Additionally, ILs tend to aggregate in solution to form amphiphilic micelles and as such display surface activity [9]. This ability to form micelles limits the number of free cations in solution and, thus, represents an upper concentration cut-off. However, they display an enhanced ability to peel off patches of lipids from the bilayer, forming new micelles of mixed composition and greater stability [67]. This affinity of ILs for biomolecules, particularly lipids, is related to the similarity of their structure and opportunity for intermolecular interactions [67].

\section{Ionic liquids against microbial biofilms}

There is a growing interest in using ILs for biofilm control, as there are no known chemical entity able to effectively kill biofilm cells and, at the same time, cause biofilm disruption/removal. Moreover, it is possible to inactivate a biofilm without causing its dispersal (i.e., biofilm inactivation and removal are distinct phenomena). Within this framework, some ILs have shown the ability to control biofilm growth and cause the disruption of the complex 3D structure, a phenomenon relevant to enhance antibiotic diffusion. As well as their potential dual action as antimicrobial and antibiofilm agents, ILs have also been reported for their plasticizing effects on surface materials commonly used in the production of functionalized medical devices with antiadhesive properties $[42,70]$.

Table 1 provides examples of ILs used in biofilm control studies. Imidazolium-based ILs are the systems most frequently used in biofilm control $[58,60,61]$, although some research has highlighted the applicability of other ILs with different cationic heads, such as quinolinium, pyridinium, phosphonium, and choline. Generally, the antibiofilm performance of ILs is modulated by the length of the tail, increasing with the increase of the alkyl chain length [71].

Compounds from a library of 1-alkyl-3methylimidazolium chloride ILs showed significant antibiofilm activity against a range of clinically important Gram-positive bacteria, with 1-decyl-3-methylimidazolium chloride being highlighted for the strongest activity [31]. From a library of quinolone-based ILs, 1-alkylquinolinium bromide salts with 12 and 14 carbons in the alkyl chain demonstrated strong antibiofilm effects against diverse bacterial pathogens [34]. Imidazolium-based monocationic and dicationic ILs with an anthracene moiety covalently attached to the imidazolium efficiently inhibited the biofilms of Gram-positive bacteria [69] Furthermore, a recent study reported the synergistic bactericidal and antibiofilm activities of 1alkyl-3-methylimidazolium ILs, with the incorporation of anions containing silver or copper, against Pseudomonas aeruginosa and Staphylococcus aureus [39].

Pyridinium ILs, including bisalkylpyridinium derivatives, were proposed to be used as topical agents to control biofilm infections [48]. Choi et al. reported the dual functional of ILs where 1-ethylpyridinium docusate and tributyl(2hydroxyethyl)phosphonium docusate were proposed for the decontamination of hospital 
TABLE 1

Examples of ILs with relevant antibiofilm activity.

IL family Compound(s)

1-Hexadecyl-3-methylimidazolium chloride

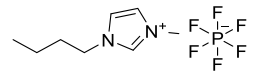

1-Butyl-3-methylimidazolium hexafluorophosphate (incorporated in polymeric nanoparticles)

$$
\mathrm{T}_{2} \mathrm{~N}^{-\mathrm{N}^{+}-}
$$

1-Octyl-3-methylimidazolium chloride<smiles>Cn1cccc1-n1cccc2cc3ccccc3c1-2</smiles>

(Anthracen-9-ylmethyl)-1-methyl-1H-imidazol-3-ium bromide

Quinoline

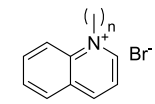

1-Alkylquinolinium bromides with variable length chain

$$
(n=8,10,12,14)
$$

Pyridine

\begin{tabular}{lll}
\hline Microorganism & Effects & Refs \\
\hline Candida albicans & Biofilm inhibition & {$[75]$}
\end{tabular}

Biofilm inhibition<smiles>CCCCCCCCC(CC)COC(=O)C(CC(=O)OCC(CC)CCCC)C(=O)OS(=O)(=O)O</smiles>

Tributyl(2-hydroxyethyl)phosphonium docusate

Choline

$$
\mathrm{N}^{+} \mathrm{OH}_{\mathrm{O}} \mathrm{O}
$$$$
\text { Choline-geranate }
$$

Dialkylamine

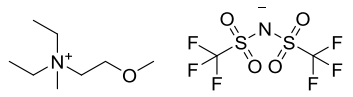

$\mathrm{N}, \mathrm{N}$-Diethyl- $\mathrm{N}$-methyl- $\mathrm{N}$-(2-methoxyethyl)ammonium bis(trifluoro methanesulfonyl)imide (for polymer brush coating)

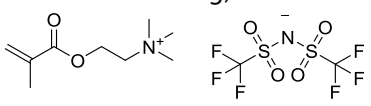

$\mathrm{N}, \mathrm{N}$-Diethyl- $\mathrm{N}$-(2-methancryloylethyl)- $\mathrm{N}$-methylammonium bis(trifluoromethylsulfonyl)imide (for polymer brush coating)
Candida tropicalis Navicula sp.

Staphylococcus epidermidis

Staphylococcus aureus

S. epidermidis, Staphylococcus haemolyticus

S. epidermidis, Pseudomonas aeruginosa, Klebsiella aerogenes, Bacillus cereus

Coagulase-negative staphylococci (CoNS)

P. aeruginosa, S. aureus

Methicillin-resistant S. aureus

Prevention of microbial adhesion and biofilm

formation

Biofilm inhibition

Biofilm disruption

Biofilm inhibition

Biofilm inhibition

Biofilm inhibition

Prevention of adhesion and biofilm inhibition

Biofilm inhibition

P. aeruginosa, Salmonella enterica Biofilm inhibition

Escherichia coli, S. epidermidis

Biofilm dispersal 
surfaces, because they act as both antimicrobial and antibiofilm-forming agents [73].

Current trends in ILs research highlight choline-based ILs as promising for the control of pathogenic biofilms. In particular, choline geranate demonstrated strong effects against biofilms of Salmonella enterica and Pseudomonas aeruginosa, and an increased delivery of the antibiotic cefadroxil by $>16$-fold into the deep tissue layers of the skin without inducing skin irritation [41]. The in vivo efficacy of choline geranate was further validated in a $P$. aeruginosa biofilm-infected wound model, proposing the use of ILs for simultaneous enhancement of topical drug delivery and antibiotic activity. More recently, innovative $\mathrm{N}$-alkylimidazolium ILs functionalized with plant secondary metabolites (phytochemicals) of the shikimate pathway, such as $p$-coumaric and cinnamic acids, and with different chain lengths, were developed. They demonstrated antibiofilm activity against Gramnegative and Gram-positive bacteria, with the strongest potency being found for derivatives that contained alkyl chains of eight and ten carbons [74]. 1-Cinnamoyl-3-octyl-1H-imidazol3-ium bromide was revealed to be the strongest IL [74]. The same strategy has been implemented using amino acids as modifiers of the imidazolium cation [70].

\section{Concluding remarks}

ILs are remarkable chemical compounds with diverse pharmaceutical and biotechnological applications. Given their broad chemical space and highly tunable nature, they are relevant players in drug discovery and drug delivery projects. With the drugdiscovery pipeline currently clogged, ILs could be exploited as an interesting strategy to tackle pathogens. Current knowledge highlights the potential of ILs to control microbial growth in both planktonic and biofilm states. However, even if ILs are promising for biofilm control, through the inactivation of the colonizer cells and the disruption of the extracellular polymeric ties, further studies are needed to understand their mode of action, toxicity, and metabolic profile, and biodegradation.

\section{Conflict of interests}

None declared.

\section{Acknowledgments}

Authors acknowledge the financial support from: Base Funding - UIDB/00511/2020 of LEPABE, UIDB/00081/2020 of CIQUP, and UIDB/ $04469 / 2020$ of CEB funded by national funds through the FCT/MCTES (PIDDAC); Project
Biocide_for_Biofilm - PTDC/BII-BTI/30219/2017 POCI-01-0145-FEDER-030219, ABFISH - PTDC/ ASP-PES/28397/2017 - POCI-01-0145-FEDER028397, funded by FEDER funds through COMPETE2020 - Programa Operacional Competitividade e Internacionalização (POCI) and by national funds (PIDDAC) through FCT/ MCTES, and BioTecNorte operation (NORTE-010145-FEDER-000004) funded by the European Regional Development Fund under the scope of Norte2020 - Programa Operacional Regional do Norte.

\section{References}

1 Donlan, R.M. et al. (2002) Biofilms: survival mechanisms of clinically relevant microorganisms. Clin. Microbiol. Rev. 15, 167-193

2 Hall-Stoodley, L. et al. (2004) Bacterial biofilms: from the natural environment to infectious diseases. Nat. Rev. Microbiol. 2, 95-108

3 Das, S. and Kumas, S. et al. (2019) Biofilms in human health. In Biofilms in Human Diseases: Treatment and Control (Kumas, S., ed.), pp. 27-43, Springer International Publishing

4 Harro, J.M. et al. (2020) Development of a novel and rapid antibody-based diagnostic for chronic Staphylococcus aureus infections based on biofilm antigens. J. Clin. Microbiol. 58, 1414-1419

5 Macià, M.D. et al. (2014) Antimicrobial susceptibility testing in biofilm-growing bacteria. Clin. Microbiol. Infect. 20, 981-990

6 Eiff, C.V. et al. (2005) Infections associated with medical devices pathogenesis, management and prophylaxis. Drugs 65, 179-214

7 Simões, M. (2012) Antimicrobial strategies effective against infectious bacterial biofilms. Curr. Med. Chem. 18, 2129-2145

8 McDonnell, G. and Russell, A.D. (1999) Antiseptics and disinfectants: activity, action, and resistance. Clin. Microbiol. Rev. 12, 147-179

9 Pendleton, J.N. et al. (2015) The antimicrobial potential of ionic liquids: a source of chemical diversity for infection and biofilm control. Int. J. Antimicrob. Agents 46, 131-139

10 Duman, A.N. et al. (2019) Synthesis of new watersoluble ionic liquids and their antibacterial profile against Gram-positive and Gram-negative bacteria. Heliyon 5, e02607

11 Elshaarawy, R.F.M. and Janiak, C. (2014) Toward new classes of potent antibiotics: synthesis and antimicrobial activity of novel metallosaldachimidazolium salts. Eur. J. Med. Chem. 75, 31-42

12 Elshaarawy, R.F.M. et al. (2014) New water soluble bisimidazolium salts with a Saldach scaffold: synthesis, characterization and in vitro cytotoxicity/bactericidal studies. Inorg. Chim. Acta 421, 110-122

13 Elshaarawy, R.F.M. and Janiak, C. (2014) Ionic liquidsupported chiral saldach with tunable hydrogen bonding: synthesis, metalation with Fe(III) and in vitro antimicrobial susceptibility. Tetrahedron 70, 80238032

14 Anastas, P.T. and Warner, J.C. (1998) Green Chemistry: Theory and Practice. Oxford University Press

15 Egorova, K.S. et al. (2017) Biological activity of ionic liquids and their application in pharmaceutics and medicine. Chem. Rev. 117, 7132-7189
16 Elshaarawy, R.F.M. et al. (2016) Pharmacological performance of novelpoly-(ionic liquid)-grafted chitosan-N-salicylidene Schiff bases and their complexes. Carbohydr. Polym. 146, 376-387

17 Garcia, H. et al. (2010) Dissolution of cork biopolymers in biocompatible ionic liquids. Green. Chem. 12 367-336

18 Hodyna, D. et al. (2018) Imidazolium ionic liquids as effective antiseptics and disinfectants against drug resistant S. aureus: In silico and in vitro studies. Comput. Biol. Chem. 73, 127-138

19 Wasserscheid, P. (2006) Volatile times for ionic liquids. Nature 439, 797

20 Seter, M. et al. (2012) Dual active ionic liquids and organic salts for inhibition of microbially influenced corrosion. Chem. Commun. 48, 5983-5985

21 Marrucho, I.M. et al. (2014) lonic liquids in pharmaceutical applications. Annu. Rev. Chem. Biomol. Eng. 5, 527-546

22 Singh, S.K. et al. (2020) lonic liquids synthesis and applications: an overview. J. Mol. Liq. 297, 112038

23 MacFarlane, M.R. (2007) lonic liquids in electrochemical devices and processes: managing interfacial electrochemistry. Acc. Chem. Res. 40, 1165-1173

24 Welton, T. (2018) lonic liquids: a brief history. Biophysical Rev. 10, 691-706

25 Miskiewicz, A. et al. (2018) The use of liquids ionic fluids as pharmaceutically active substances helpful in combating nosocomial infections induced by Klebsiella pneumoniae New Delhi strain, Acinetobacter baumannii and Enterococcus species. Int. J. Mol. Sci. 19, 2779

26 Agatemor, C. et al. (2018) lonic liquids for addressing unmet needs in healthcare. Bioeng. Transl. Med. 3, 7-25

27 Gadilohar, B.L. et al. (2017) Choline based ionic liquids and their applications in organic transformation. J. Mol. Liq. 227, 234-261

28 Florio, W. et al. (2019) Comparative evaluation of antimicrobial activity of different types of ionic liquids. Mater. Sci. Eng. C 104, 109907

29 Demberelnyamba, D. et al. (2004) Synthesis and antimicrobial properties of imidazolium and pyrrolidinonium salts. Bioorg. Med. Chem. 12, 853-857

30 Iwai, N. et al. (2011) Antibacterial activities of imidazolium, pyrrolidinium and piperidinium salts. Bioorg. Med. Chem. Lett. 21, 1728-1730

31 Gilmore, B.F. (2011) Antimicrobial ionic liquids. In Ionic Liquids: Applications and Perspectives (Kokorin, A., ed.), pp. 587-604, IntechOpen

32 Diallo, A.O. et al. (2012) Revisiting physico-chemical hazards of ionic liquids. Sep. Purif. Technol. 97, 228-234

33 Pernak, J. et al. (2007) Synthesis and properties of trigeminal tricationic ionic liquids. Chemistry 13, 31063112

34 Busetti, A. et al. (2010) Antimicrobial and antibiofilm activities of 1-alkylquinolinium bromide ionic liquids. Green. Chem. 12, 420-425

35 Cole, M.R. et al. (2011) Design, synthesis, and biological evaluation of $\beta$-lactam antibiotic-based imidazoliumand pyridinium-type ionic liquids. Chem. Biol. Drug. Des. 78, 33-41

36 Sekhon, B.S. (2011) lonic liquids: pharmaceutical and biotechnological applications. Asian J. Pharm. Biol. Res. 1, 395-411

37 Łuczak, J. et al. (2010) Antimicrobial and surface activity of 1-alkyl-3-methylimidazolium derivatives. Green Chem. 12, 593-601

38 Garcia, M.T. et al. (2014) Self-assembly and antimicrobial activity of long-chain amide- 
functionalized ionic liquids in aqueous solution. Colloids Surf. B 123, 318-325

39 Gilmore, B.F. et al. (2013) Enhanced antimicrobial activities of 1-alkyl-3-methyl imidazolium ionic liquids based on silver or copper containing anions. New J. Chem. 37, 873-876

40 Cieniecka-Rosłonkiewicz, A. et al. (2005) Synthesis, anti-microbial activities and anti-electrostatic properties of phosphonium-based ionic liquids. Green Chem. 7, 855-862

41 Mester, P. et al. (2015) Antimicrobial effects of short chained imidazolium-based ionic liquids-Influence of anion chaotropicity. Ecotoxicol. Environ. Saf. 111, 96-101

42 Zakrewsky, M. et al. (2014) Ionic liquids as a class of materials for transdermal delivery and pathogen neutralization. Proc. Natl. Acad. Sci. U. S. A. 111, 13313 13318

43 Sydow, M. et al. (2018) Biodiversity of soil bacteria exposed to sub-lethal concentrations of phosphonium-based ionic liquids: effects of toxicity and biodegradation. Ecotoxicol. Environ. Saf. 147, 157-164

44 Guo, J. et al. (2015) Intrinsically antibacterial poly(ionic liquid) membranes: the synergistic effect of anions. ACS Macro. Lett. 4, 1094-1098

45 Hoque, J. et al. (2016) Antibacterial and antibiofilm activity of cationic small molecules with spatia positioning of hydrophobicity: an in vitro and in vivo evaluation. J. Med. Chem. 59, 10750-10762

46 Seter, M. et al. (2012) Dual active ionic liquids and organic salts for inhibition of microbially influenced corrosion. Chem. Commun. 48, 5983-5985

47 Nusaibah, A.M. et al. (2016) A review on dicationic ionic liquids: classification and application. Ind. Eng. Manag. 5, 1-7

48 O'Toole, G.A. et al. (2012) Diphosphonium ionic liquids as broad-spectrum antimicrobial agents. Cornea 31 , $810-816$

49 Huang, W. et al. (2020) lonic liquids: green and tailor made solvents in drug delivery. Drug Discov. Today 25 901-908

50 Williams, H.D. et al. (2014) lonic liquids provide unique opportunities for oral drug delivery: structure optimization and in vivo evidence of utility. Chem. Commun. 50, 1688-1690

51 Ventura, S.P.M. et al. (2013) Designing ionic liquids: the chemical structure role in the toxicity. Ecotoxicology 22 $1-12$

52 Sidat, Z. et al. (2019) Ionic liquids as potential and synergistic permeation enhancers for transdermal drug delivery. Pharmaceutics 11, 96

53 Liu, J. et al. (2020) Novel 2-substituted 3-hydroxy-1,6dimethylpyridin- $4(1 \mathrm{H})$-ones as dual-acting biofilm inhibitors of Pseudomonas aeruginosa. J. Med. Chem. 63, 10921-10945

54 Yao, W. et al. (2016) Tuning the hydrophilicity and hydrophobicity of the respective cation and anion: reversible phase transfer of ionic liquids. Angew. Chem. Int. Ed. 55, 7934-7938

55 Hanna, S.L. et al. (2017) Synergistic effects of polymyxin and ionic liquids on lipid vesicle membrane stability and aggregation. Biophys. Chem. 227, 1-7
56 Smiglak, M. et al. (2014) lonic liquids for energy, materials, and medicine. ChemComm 9228-9250

57 Nuñez-Figueredo, Y. et al. (2019) Influence of ionic liquid on graphite/silver nanoparticles dispersion and antibacterial properties against Escherichia coli of PP/ EPDM composite coatings. J. Appl. Polym. Sci. 48714, $1-10$

58 Aditya, A. et al. (2018) Biological and medical applications of materials and interfaces zinc oxide nanoparticles dispersed in ionic liquids show high antimicrobial efficacy to skin-specific bacteria. ACS Appl. Mater. Interfaces 10, 15401-15411

59 Łuczak, J. et al. (2016) Ionic liquids for nano- and microstructures preparation. Part 1: properties and multifunctional role. Adv. Colloid Interface Sci. 230 $13-28$

60 De Almeida, T.S. et al. (2017) An emerging integration between ionic liquids and nanotechnology: general uses and future prospects in drug delivery. Ther. Deliv. 8, 461-473

61 Rai, M. et al. (2009) Silver nanoparticles as a new generation of antimicrobials. Biotechnol. Adv. 27, 76-83

62 Brunel, F. et al. (2016) Antibacterial activities of fluorescent nano assembled triphenylamine phosphonium ionic liquids. Bioorg. Med. Chem. Lett. 26, 3770-3773

63 Kumari, P. et al. (2020) Mechanisms of action of ionic liquids on living cells: the state of the art. Biophys. Rev. $12,1187-1215$

64 Samorì, C. (2011) Ionic liquids and their biological effects towards microorganisms. Curr. Org. Chem. 15 , 1888-1904

65 Kenawy, E.-R. and Kandil, S. et al. (2013) Synthesis, antimicrobial activity and applications of polymers with Ammonium and phosphonium groups. In Polymeric Materials with Antimicrobial Activity: From Synthesis to applications (Muñoz-Bonilla, A., ed.), pp. 54-74, Royal Society for Chemistry

66 Hodyna, D. et al. (2016) Efficient antimicrobial activity and reduced toxicity of 1-dodecyl-3methylimidazolium tetrafluoroborate ionic liquid/ $\beta$-cyclodextrin complex. Chem. Eng. J. 284, 1136-1145

67 Benedetto, A. et al. (2018) Room-temperature ionic liquids and biomembranes: setting the stage for applications in pharmacology, biomedicine, and bionanotechnology. Langmuir 34, 9579-9597

68 Arning, J. et al. (2008) Qualitative and quantitative structure activity relationships for the inhibitory effects of cationic head groups, functionalised side chains and anions of ionic liquids on acetylcholinesterase. Green Chem. 10, 47-58

69 Jha, G. et al. (2017) Synthesis, photophysical studies on some anthracene-based ionic liquids and their application as biofilm formation inhibitor. Chem. Eur. 2, $2426-2432$

70 Egorova, K.S. et al. (2018) Fundamental importance of ionic interactions in the liquid phase: a review of recent studies of ionic liquids in biomedical and pharmaceutical applications. J. Mol. Liq. 272, 271 300

71 Coleman, D. et al. (2012) Antimicrobial toxicity studies of ionic liquids leading to a 'hit' MRSA selective antibacterial imidazolium salt. Green Chem. 14, 13501356

72 Carson, L. et al. (2009) Antibiofilm activities of 1-alkyl-3methylimidazolium chloride ionic liquids. Green Chem. 44, 492-497

73 Choi, S.Y. et al. (2011) Dual functional ionic liquids as plasticisers and antimicrobial agents for medical polymers. Green Chem. 13, 1527-1535

74 Forero-Doria, O. et al. (2019) N-alkylimidazolium salts functionalized with $p$-coumaric and cinnamic acid: a study of their antimicrobial and antibiofilm effects. Molecules 24, 3484

75 Reddy, G.K.K. et al. (2020) Alkylimidazolium ionic liquids as antifungal alternatives: antibiofilm activity against Candida albicans and underlying mechanism of action. Front. Microbiol. 11, 1-15

76 Bergamo, V.Z. et al. (2015) Imidazolium salts as antifungal agents: strong antibiofilm activity against multidrug-resistant Candida tropicalis isolates. Lett. Appl. Microbiol. 60, 66-71

77 Reddy, G.K.K. et al. (2017) Long alkyl-chain imidazolium ionic liquids: antibiofilm activity against phototrophic biofilms. Colloids Surf. B. 155, 487-496

78 Takahashi, C. et al. (2019) Optimization of ionic liquidincorporated PLGA nanoparticles for treatment of biofilm infections. Mater. Sci. Eng. C 97, 78-83

79 Wylie, M.P. et al. (2020) Phosphonium ionic liquidinfused poly(vinyl chloride) surfaces possessing potent antifouling properties. ACS Omega 5, 7771-7781

80 Kanematsu, H. et al. (2018) Biofilm formation of a polymer brush coating with ionic liquids compared to a polymer brush coating with a non-ionic liquid. Coatings 8,398

\section{Manuel Simões ${ }^{1, *}$ \\ Ana Rita Pereira ${ }^{1}$ \\ Lúcia Chaves Simões ${ }^{2}$ \\ Fernando Cagide ${ }^{3}$ \\ Fernanda Borges ${ }^{3, *}$}

'LEPABE, Department of Chemical Engineering, Faculty of Engineering, University of Porto, 4200-465 Porto, PortugalLEPABE, Department of Chemical Engineering, Faculty of Engineering, University of Porto, 4200-465 Porto, Portugal ${ }^{2} C E B$, Centre of Biological Engineering, University of Minho, Campus de Gualtar 4710-057 Braga, PortugalCEB, Centre of Biological Engineering, University of Minho, Campus de Gualtar 4710057 Braga, Portugal

${ }^{3}$ CIQUP, Department of Chemistry and Biochemistry, Faculty of Sciences, University of Porto, 4169007 Porto, PortugalCIQUP, Department of Chemistry and Biochemistry, Faculty of Sciences, University of Porto, 4169-007 Porto, Portugal

\section{*Corresponding authors:}

